Mittlere Örter der Vergleichsterne.

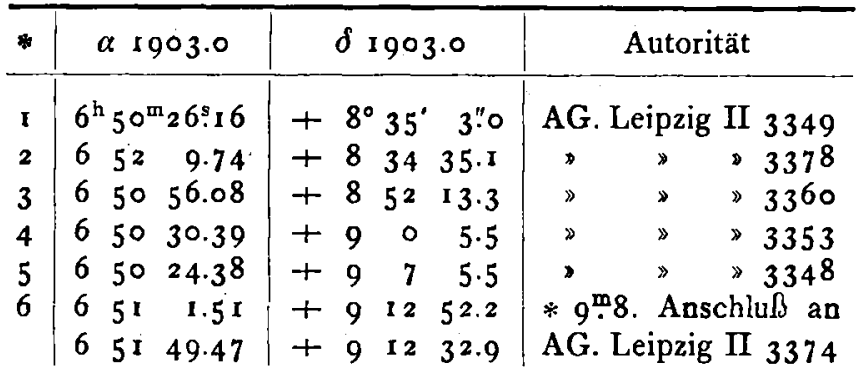

\begin{tabular}{|c|c|c|c|}
\hline * & $\alpha 1903.0$ & $\delta 1903.0$ & Autorität \\
\hline 7 & $6^{\mathrm{h}} 49^{\mathrm{m}} \quad 7^{\mathrm{s}} \cdot 20$ & $+9^{\circ} 34^{\prime} 36.0$ & AG. Leipzig II 3318 \\
\hline 8 & $\begin{array}{lll}6 & 49 & 14.96\end{array}$ & $+932 \times 7.4$ & $\star \quad \otimes 3321$ \\
\hline 9 & 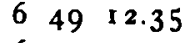 & $+93^{627.5}$ & $\gg 3320$ \\
\hline Io & $\begin{array}{ll}6 & 49.34 .79\end{array}$ & $+937 \times 8.3$ & $\nabla 3327$ \\
\hline I I & $\begin{array}{lll}6 & 49 & 0.89\end{array}$ & $+1010 \quad 3.9$ & AG. Leipzig I 257 I \\
\hline 12 & $\begin{array}{lll}6 & 43 & 39.90\end{array}$ & +132840.2 & $\times 2509$ \\
\hline
\end{tabular}

Vergleichung der Beobachtungen mit der Ephemeride von Dr. Ristenpart in A.N. $3^{8} 3^{8}$ (B-R).

\begin{tabular}{|c|c|c|}
\hline 1903 & $\Delta \alpha$ & $\Delta \delta$ \\
\hline Jan. 16 & +1.79 & $-2^{\prime} \mathrm{I} 6^{\prime \prime} \mathrm{I}$ \\
\hline 16 & +1.92 & $\begin{array}{ll}-2 & 8.6\end{array}$ \\
\hline 17 & +2.11 & -22 I. 2 \\
\hline 17 & +2.33 & $-2 \quad 21.9$ \\
\hline
\end{tabular}

\begin{tabular}{c|c|c}
\hline \multicolumn{1}{c|}{1903} & $\Delta \alpha$ & $\Delta \delta$ \\
\hline Jan. 18 & +2.83 & -2 \\
18 & +2.47 & -227.5 \\
19 & +2.99 & -234.7 \\
19 & +2.96 & -233.4
\end{tabular}

\begin{tabular}{|c|c|c|}
\hline 1902 & $\Delta \alpha$ & $\Delta \delta$ \\
\hline Jan. $\quad 19$ & +2.53 & $-2^{\prime} 33^{\prime \prime} \cdot 9$ \\
\hline 19 & +2.94 & $\begin{array}{ll}-2 & 34.4\end{array}$ \\
\hline 21 & +3.02 & -249.2 \\
\hline 31 & $+5 \cdot 5^{8}$ & $\begin{array}{ll}-4 & 5 \cdot 4\end{array}$ \\
\hline
\end{tabular}

Bemerkungen.

Jan. I6. Beobachtung durch Wolken mehrfach unter- helligkeit $1 \mathbf{x}^{\mathrm{m}} \cdot \mathrm{o}$. Jan. $2 \mathrm{I}$. Dunstige Atmosphäre, Komet brochen. $\Delta \delta$ sehr unsicher. - Jan. I 7 und 19. Komet er- kaum zu sehen; Fadenbeleuchtung setzt schließlich aus. scheint als ein runder zarter Nebel mit Kern $12^{m} \cdot 5$, Gesamt- Jan. 31 . Luft klar, aber sehr unruhig.

Hamburg, 1903 Febr. 5 .

K. Graff.

\title{
Osservazioni della cometa 1903 a
}

fatte all' equatoriale di $39 \mathrm{~cm}$ del R. Osservatorio astronomico al Collegio Romano.

Seguito, vedi A. N. 3844 .

\begin{tabular}{|c|c|c|c|c|c|c|c|c|c|c|c|c|c|c|c|c|c|c|}
\hline \multirow{3}{*}{$\begin{array}{l}1903 \\
\text { Febb.19 } \\
\text { Marz. } 6\end{array}$} & \multicolumn{2}{|c|}{ T. m. Roma } & \multicolumn{2}{|r|}{$\Delta \alpha$} & \multicolumn{2}{|c|}{$\Delta d$} & \multirow{2}{*}{$\frac{\text { Cf. }}{26.9}$} & \multirow{2}{*}{$\frac{\text { Oss. }}{\mathrm{M}}$} & \multicolumn{3}{|c|}{$\alpha$ app. } & \multirow{2}{*}{$\frac{\log p \cdot \Delta \mid}{9.640 \mid}$} & \multicolumn{2}{|c|}{$\delta$ app. } & \multirow{2}{*}{$\frac{\log p \cdot A \mid}{0.73^{6}}$} & \multicolumn{2}{|c|}{ Red. ad 1. app. } & * \\
\hline & $6^{b} 5^{8}$ & $0^{5}$ & - & $0^{m} 10^{5} \cdot 52$ & + & $I^{\prime} 40 . .2$ & & & $23^{k}$ & $41^{\mathrm{m}}$ & 26.55 & & $+11^{\circ} 36$ & 0.0 & & -0.22 & $+0 . .9$ & I \\
\hline & 72 & 6 & + & $0 \quad 13.09$ & $-I C$ & O II.I & 22.3 & $\mathbf{M}$ & 0 & I I & 12.20 & 9.659 & +1651 & 17.6 & 0.737 & -0.15 & -0.2 & 2 \\
\hline IO & $65 \mathrm{I}$ & 29 & - & 426.84 & +3 & 322.1 & $9 \cdot 3$ & $\mathbf{M}$ & 0 & I 8 & $3^{2} \cdot 36$ & 9.660 & +1724 & $4^{2.2}$ & 0.734 & -0.16 & -0.7 & 3 \\
\hline II & 70 & 3 & - & 250.06 & + & $\begin{array}{lll}2 & 1 & 1\end{array}$ & 12.3 & B & $\circ$ & 20 & 9.14 & $9.66 \mathrm{I}$ & +1723 & $3 r \cdot 3$ & 0.742 & -0.16 & -0.8 & 3. \\
\hline 13 & 647 & 25 & - & 143.43 & + & 53.8 & I 2.4 & $\mathbf{M}$ & 0 & 22 & $5^{6.73}$ & 9.659 & +175 & 20.8 & $0.73^{8}$ & -0.13 & -1.0 & 4 \\
\hline 13 & $7 \quad I$ & $4 I$ & - & I 42.65 & + & 45.9 & 12.4 & B & 0 & 22 & $57.5 \mathrm{I}$ & 9.660 & +17 & I 2.9 & 0.747 & $\gg$ & , & 4 \\
\hline I 4 & 7 & $5 \mathrm{I}$ & -2 & 237.05 & + & I 53.4 & 21.7 & B & 0 & 24 & $7 \cdot 43$ & 9.659 & +1646 & 34.7 & 0.753 & -0.13 & $-I .2$ & 5 \\
\hline 18 & 7 & $4^{r}$ & -1 & 78.05 & - & 329.3 & 3.1 & $\mathbf{M}$ & 0 & 26 & 51.66 & 9.652 & +1413 & 16.5 & 0.760 & -0.12 & -2.1 & 6 \\
\hline
\end{tabular}

Posizioni medie delle stelle di confronto.

\begin{tabular}{|c|c|c|c|}
\hline$*$ & $\alpha 1903.0$ & $\delta 1903.0$ & Autorità \\
\hline I & $23^{h} 4^{1^{m}} 37^{s} \cdot 29$ & $+11^{\circ} 34^{\prime}$ 1 8."9 & AG. Leipzig I $943^{6}$ \\
\hline 2 & $010 \quad 59.26$ & +17 I 28.9 & AG. Berlin A. 47 \\
\hline 3 & $\circ 2259.3^{6}$ & +172120.8 & $\$ 109$ \\
\hline
\end{tabular}

\begin{tabular}{|c|c|c|c|}
\hline * & $\alpha 1903.0$ & $\delta 1903.0$ & Autorità \\
\hline 4 & $0^{\mathrm{h}} 24^{\mathrm{m}} 40.29$ & $+17^{\circ} 4^{\prime} 28{ }^{\prime \prime} \circ$ & AG. Berlin A. 119 \\
\hline 5 & 02644.48 & +164441.3 & $\gg \quad 138$ \\
\hline 6 & $\begin{array}{lll}\circ & 43 & 59.83\end{array}$ & +141647.9 & AG. Leipzig I 217 \\
\hline
\end{tabular}

Febbrajo 19, nucleo $8^{\mathrm{va}}$, nebulosità $40^{\mathrm{m}}$ diametro. - Marzo 6, nucleo $6-7^{\mathrm{ma}}$ con piccola e debole coda. Marzo 18, cometa lucentissima, ma assai bassa; la stella AG. Leipzig I 217 variabile, grandezza stimata intorno alla $6^{\text {ta }}$; BD. 6.8, Leipzig 8.0-8.2.*)

Le osservazioni vennero fatte col micrometro filare; la lettera B corrisponde al Dr. Emilio Bianchi, nuovo assistente del Osservatorio.

Roma, I 903 marzo 19.

E. Millosevich.

*) In der Potsdamer Photom. DM. ist der Stern mit der Größe $7^{\mathrm{m}} \cdot \mathrm{o}, \mathrm{F}$ arbe WG beobachtet. $K r$. 